\title{
Heavy Quark Production at HERA
}

\author{
Andreas B. Meyer ${ }^{1}$ \\ (on behalf of the H1 and ZEUS Collaborations) \\ University of Hamburg \\ II. Institut für Experimentalphysik \\ Luruper Chaussee 149 \\ D 22761 Hamburg \\ E-mail:Andreas.Meyer@desy.de
}

\begin{abstract}
Recent results on heavy flavor production at HERA are presented. New measurements of charm production are available in the region of $Q^{2}<1000 \mathrm{GeV}^{2}$ for $D^{*}$ and in the photoproduction limit, $Q^{2} \rightarrow 0$, for $D_{s}$. The results are compared to theoretical calculations and the charm proton structure function $F_{2}^{c \bar{c}}\left(x, Q^{2}\right)$ is extracted. A new independent measurement of the $b \bar{b}$ cross section using a $b$-lifetime tag is reported.
\end{abstract}

\section{INTRODUCTION}

At the storage ring HERA positrons of $27.5 \mathrm{GeV}$ collide with protons of $920 \mathrm{GeV}$ resulting in a center of mass energy $\sqrt{s}=320 \mathrm{GeV}$. The event kinematics of $e p$ scattering is described by $s=k+P, Q^{2}=-\left(k-k^{\prime}\right)^{2}, y=q \cdot P / k \cdot P, x=Q^{2} / s y$ and $W_{\gamma p}^{2}=(q+P)^{2}=s y-Q^{2}$ where $k, k^{\prime}, P$ and $q$ are the 4 -vectors of the incoming/outgoing electron, incoming proton and exchanged photon, respectively.

\section{A Heavy Flavor Production Mechanism}

Heavy quark production predominantly occurs via the process of boson gluon fusion (BGF) sketched in fig.1a. It is thus directly sensitive to the gluon distribution in the proton. At HERA the measurement of heavy flavor processes is a powerful means to study perturbative QCD down to values of $x \geq 10^{-4}$. In particular it can be tested if the parton distributions are universal, i.e. independent of the nature of the hard scattering process. The presence of two large scales, the squared 4-momentum transfer $Q^{2}$ and the heavy quark masses $m_{c, b}^{2}$ makes heavy

1) Contribution to "Workshop on Heavy Quarks at Fixed Target", Centro Brasileiro de Pesquisas Fisicas, Rio de Janeiro, Brazil, Oct. 92000 
a)

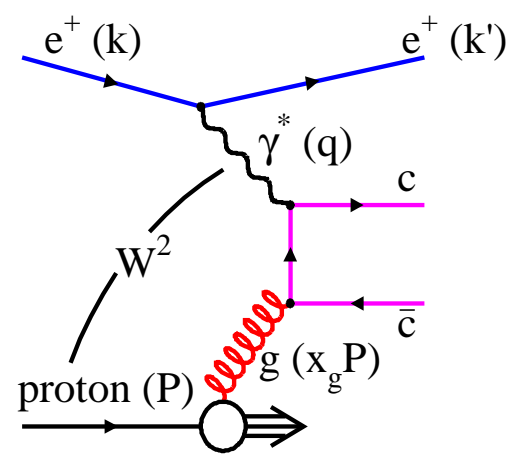

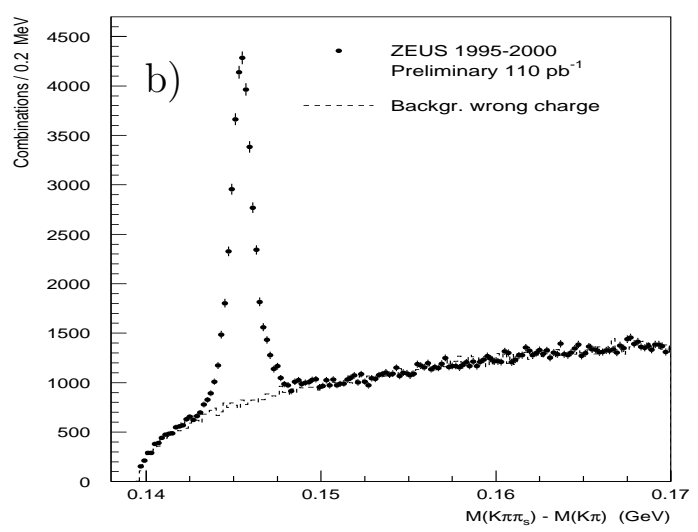

FIGURE 1. a) Charm Production via Boson-Gluon-Fusion; b) Mass difference distribution $\Delta M=M_{K \pi \pi_{s}}-M_{K \pi}$ as obtained from $110 \mathrm{pb}^{-1}$ by ZEUS.

flavor production a useful test bed for the study of perturbative QCD resummation techniques.

Several schemes to calculate heavy quark cross sections in next-to-leading order (NLO) are available. The program HVQDIS [1] implements a fixed flavor scheme at fixed order $\alpha_{s}^{2}$, assuming that the proton is made up of three active quark flavors (uds). The parton distributions are obtained by the DGLAP equation [2]. The heavy quark pairs are produced at the perturbative level via BGF. The results of HVQDIS are expected to be most accurate for $Q^{2} \sim m_{c}^{2}$. At large $Q^{2}$ terms of orders higher than $\alpha_{s}^{2}$ contain $\log \left(Q^{2} / m_{c}^{2}\right)$ factors that can become large. The CCFM equation [3] approximates the parton cascade as emitting gluons in an angular ordered way. The cross section is calculated according to the $k_{t}$-factorization theorem by convoluting an unintegrated gluon density with an off-shell BGF matrix element. A new hadron level Monte Carlo generator CASCADE [4] is available which implements the CCFM equation.
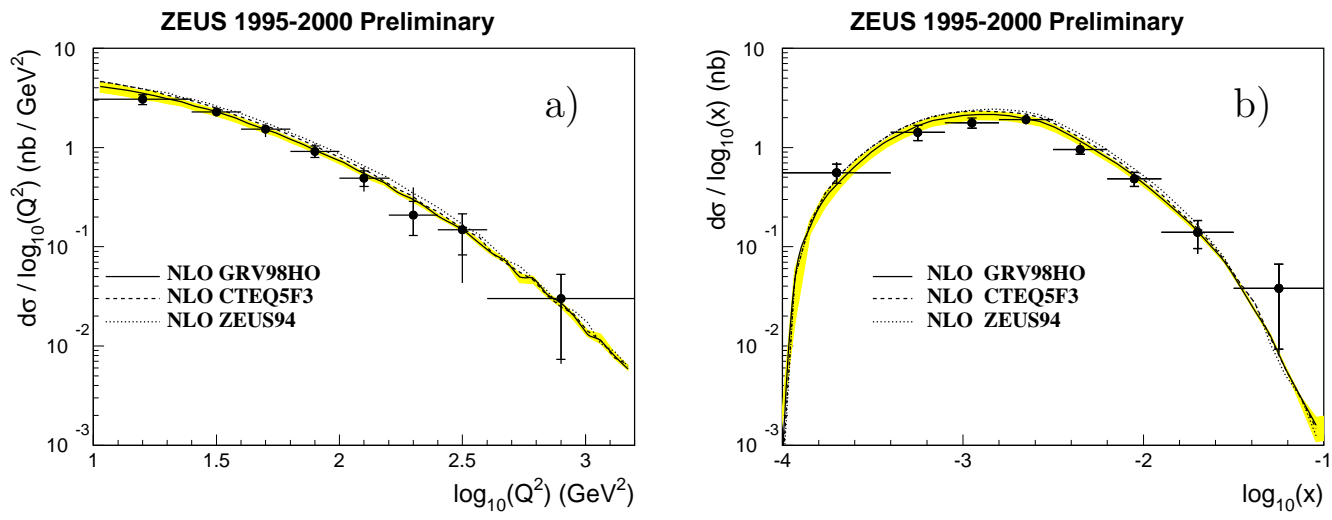

FIGURE 2. Differential $D^{*}$ production cross sections as a function of $Q^{2}(a)$ and $x(b)$ compared to the NLO QCD calculation of HVQDIS with different parton density distributions. 

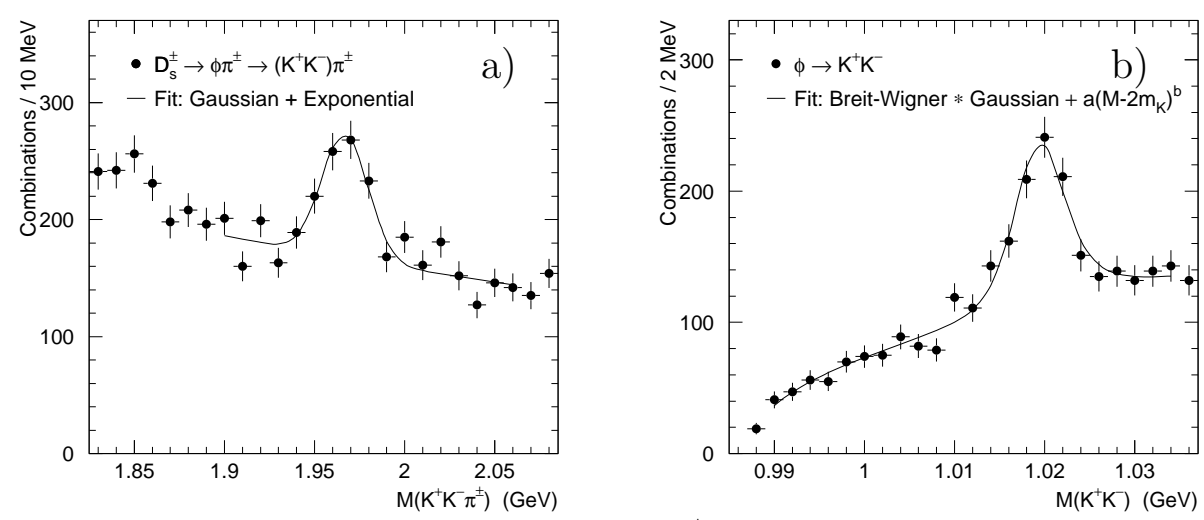

FIGURE 3. a) Distribution of $M\left(K^{+} K^{-} \pi^{ \pm}\right)$for events inside the $\phi$ mass range $1.0115<M\left(K^{+} K^{-}\right)<1.0275 \mathrm{GeV}$ as measured by ZEUS; b) $M\left(K^{+} K^{-}\right)$distribution for events inside the $D_{s}$ mass range.

\section{B Experimental Procedure}

Charm events are tagged by reconstructing $D^{*}$-mesons (H1 [5], ZEUS [6,7]), $D_{s}$ mesons (ZEUS [8]) or semi-leptonic decays into electrons (ZEUS [9]). Visible cross sections of $D$-meson production are measured in the central region of detector acceptance, at $p_{\perp}^{D}>1.5 \mathrm{GeV} / \mathrm{c}$ and $|\eta|<1.5$, where $p_{\perp}$ is the transverse momentum of the $D$-meson and $\eta$ denotes the pseudorapidity. Measurements of the $b \bar{b}$-cross section have been performed through semi-leptonic decays in muons (H1 [10], ZEUS [11]) or electrons (ZEUS [12]) and, more recently, by exploiting the long lifetime of $B$-hadrons (H1 [13]).

Since the beginning of HERA operation in 1992 the luminosity has continuously increased, yielding a total integrated luminosity collected by each of the two collider experiments $\mathrm{H} 1$ and ZEUS of more than $100 \mathrm{pb}^{-1}$. Fig.1b shows the $D^{*}-D^{0}$ mass difference distribution measured by the ZEUS experiment. There are roughly 27,000 events in the signal illustrating the excellent capabilities of HERA for the study of the charm production mechanism and also for charm meson spectroscopy studies [14]. In winter 2000/2001 the HERA accelerator is being upgraded. In the future it is expected to deliver a yearly integrated luminosity of $150 \mathrm{pb}^{-1}$.

\section{OPEN CHARM PRODUCTION}

\section{A $\quad D^{*}$ production at high $Q^{2}$}

Fig.2 shows the results of $82.6 \mathrm{pb}^{-1}$ of $D^{*}$ data collected by the ZEUS experiment [6] in the region $10<Q^{2}<1000 \mathrm{GeV}^{2}$. The shaded band represents the theoretical calculation as implemented in the HVQDIS program. Here the charm quark mass is varied between 1.3 and $1.6 \mathrm{GeV}$. The input gluon distribution is derived from 
ZEUS $96+97$

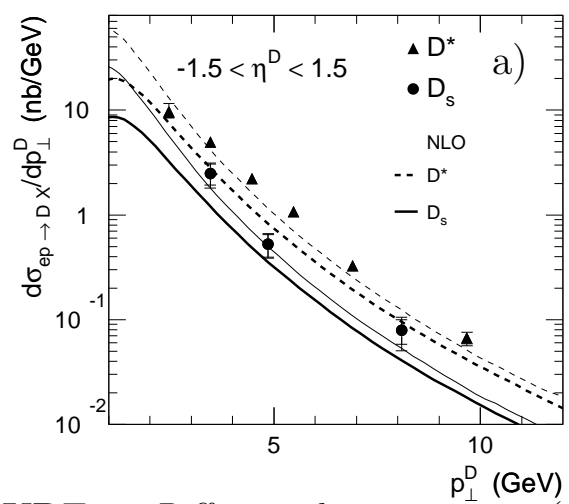

ZEUS $96+97$

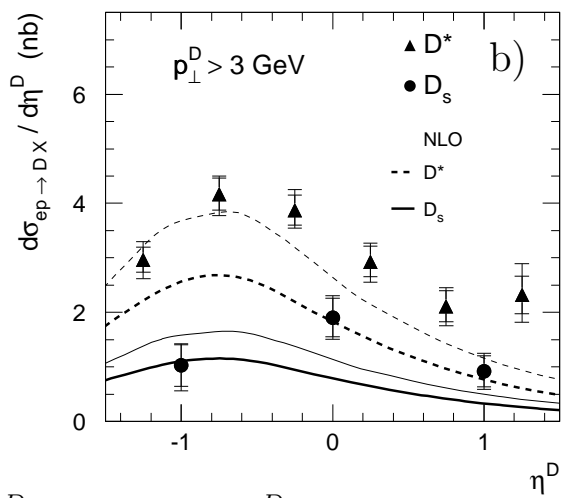

FIGURE 4. Differential cross section (a) $d \sigma / d p_{\perp}^{D}$ and (b) $d \sigma / d \eta^{D}$ for $D^{*}$ (triangles) and $D_{s}$ (bullets) in comparison with NLO calculations (see text).

results of different QCD fits to the inclusive proton structure function [15-17]. The Peterson fragmentation function [18] is used to describe the probability that a $c$ quark hadronizes into a $D^{*}$ meson. The theoretical predictions agree well with the data indicating that, within the statistics presently available, the BGF calculation in the fixed flavor scheme of order $\alpha_{s}^{2}$ fully accounts for the charm cross section up to very high values of $x$ and $Q^{2}$.

\section{B $D^{*}$ and $D_{s}$ production in photoproduction}

Visible cross sections of $D^{*}$ mesons in photoproduction have been presented before [19]. More recently a measurement of $D_{s}$-mesons was performed using the decay $D_{s} \rightarrow \phi \pi$ and $\phi \rightarrow K K$. Fig.3 shows the signals of the reconstructed $D_{s}$ and the corresponding $\phi[8]$. The visible cross section is extracted in the region $12>p_{\perp}^{D^{*}}>3$ $\mathrm{GeV}$ and $\left|\eta^{D^{*}}\right|<1.5$ yielding $\sigma_{D_{s} X}^{\text {vis }}=\left(3.79 \pm 0.59(\text { stat })_{-0.46}^{+0.26}(\right.$ syst $\left.) \pm 0.94(B R)\right) \mathrm{nb}$ where the last error accounts for the uncertainty of the branching ratio. Fig.4 compares the measured visible production cross sections to NLO predictions [20]. The bold curves refer to a charm mass value $m_{c}=1.5 \mathrm{GeV}$ and the renormalization scale $m_{R}=a \sqrt{m_{c}^{2}+p_{\perp}^{2}}$ with $a=1$. From fig.4a it is evident that the normalization of the theoretical prediction is significantly too low even for extreme values $m_{c}=1.2 \mathrm{GeV}$ and $a=0.5$ (thin lines). Furthermore the shape (fig.4b) is inconsistent with the data which are much higher in the forward direction $\eta>0$. This behavior of the data could be due to an interaction between the color charges of the $c$-quark and the proton remnant (beam drag) [21] which is not accounted for in the model. With the PYTHIA fragmentation the agreement is slightly better [22]. In the same kinematic region a result of $\sigma_{D^{*}}^{v i s}=$ $\left(9.17 \pm 0.35(\text { stat })_{-0.39}^{+0.40}(\right.$ syst $\left.)\right) \mathrm{nb}$ is obtained for $D^{*}$ production. The ratio $\sigma_{D_{s}}^{\text {vis }} / \sigma_{D^{*}}^{\text {vis }}$ is determined as $0.41 \pm 0.07$ (syst $)_{-0.05}^{+0.03}($ stat $) \pm 0.10(B R)$. This result is compared to the ratio of fragmentation probabilities $f\left(c \rightarrow D_{s}\right) / f\left(c \rightarrow D^{*}\right)=0.43 \pm 0.04 \pm 0.11$ 
ZEUS $1996-97$
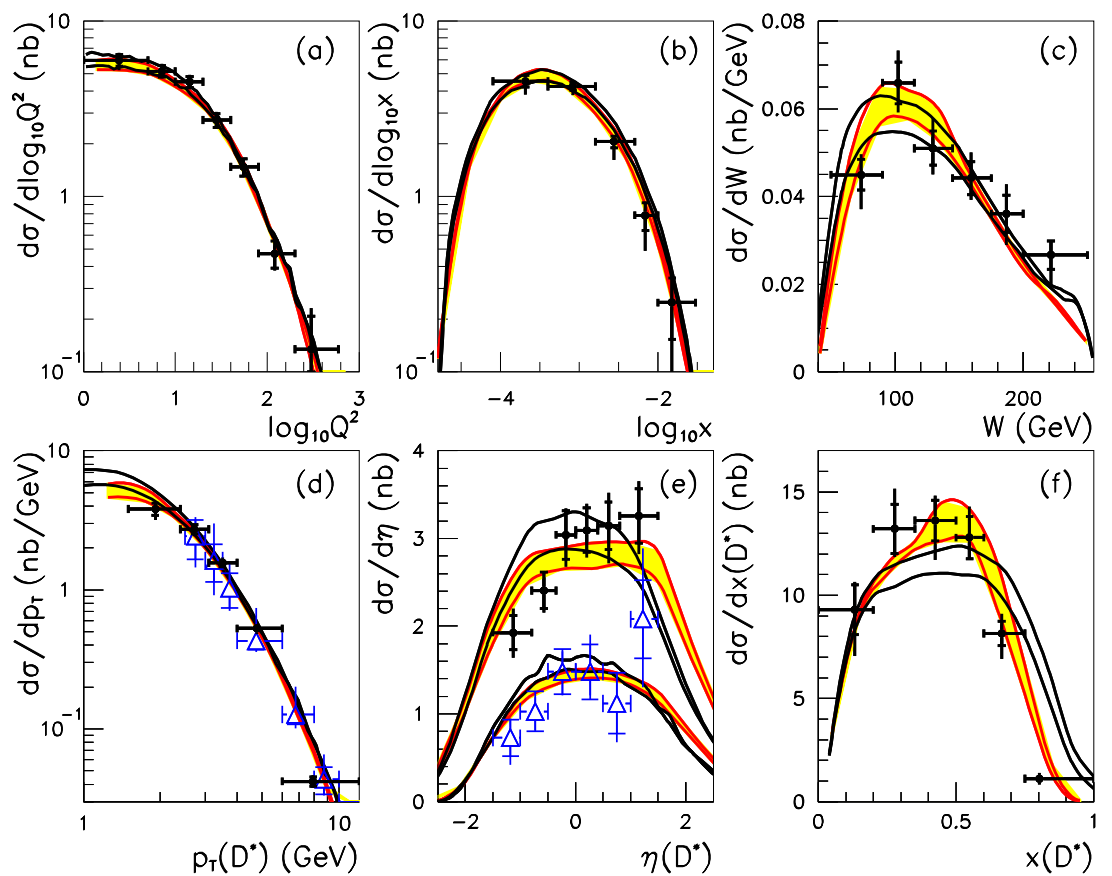

FIGURE 5. Differential cross sections for $D^{*}$ production from the $K \pi \pi$ final state (bullets).

Fig. $d$ and e also show the cross section for the $K 4 \pi$ decay channel.

as obtained from $e^{+} e^{-}$experiments [23]. The strangeness suppression factor $\gamma_{s}$ is the ratio of probabilities to create $s$ or $(u, d)$ quarks during the fragmentation process. In simulations based on the Lund String Model [24] $\gamma_{s}$ can be adjusted. For HERA an optimal value of $0.27 \pm 0.05$ is found while for $e^{+} e^{-}$experiments $0.26 \pm 0.03$ had been obtained [23]. While direct measurements of the fragmentation function at HERA are not yet available, the agreement shows a consistent picture of charm fragmentation which tends to support the universality of charm fragmentation.

\section{C $\quad D^{*}$ cross sections at small $Q^{2}$}

Visible $D^{*}$ cross sections at small values of $Q^{2}$ have been measured by both H1 [5] and ZEUS [7] yielding $\sigma_{e p \rightarrow e D^{*} X}^{\mathrm{ZEUS}}=8.31 \pm 0.31(\text { stat })_{-0.5}^{+0.3}($ syst $) \mathrm{nb}$ and $\sigma_{e p \rightarrow e D^{*} X}^{\mathrm{H} 1}=$ $8.37 \pm 0.41(\text { stat })_{-0.82}^{+1.11}(\text { syst })_{-0.39}^{+0.64}($ theo $)$ nb for slightly different kinematic regions (ZEUS: $1<Q^{2}<600 \mathrm{GeV}^{2}, y<0.7,1.5<p_{\perp}<15 \mathrm{GeV},|\eta|<1.5 ; \mathrm{H} 1: 1<Q^{2}<$ $\left.100 \mathrm{GeV}^{2}, 0.05<y<0.7, p_{\perp}>1.5 \mathrm{GeV},|\eta|<1.5\right)$. The ZEUS data are shown in fig.5 in comparison with HVQDIS. The open band corresponds to the standard Peterson fragmentation function. To account for potential beam-drag effects the Peterson fragmentation is replaced by that of the RAPGAP simulation [25] (shaded 

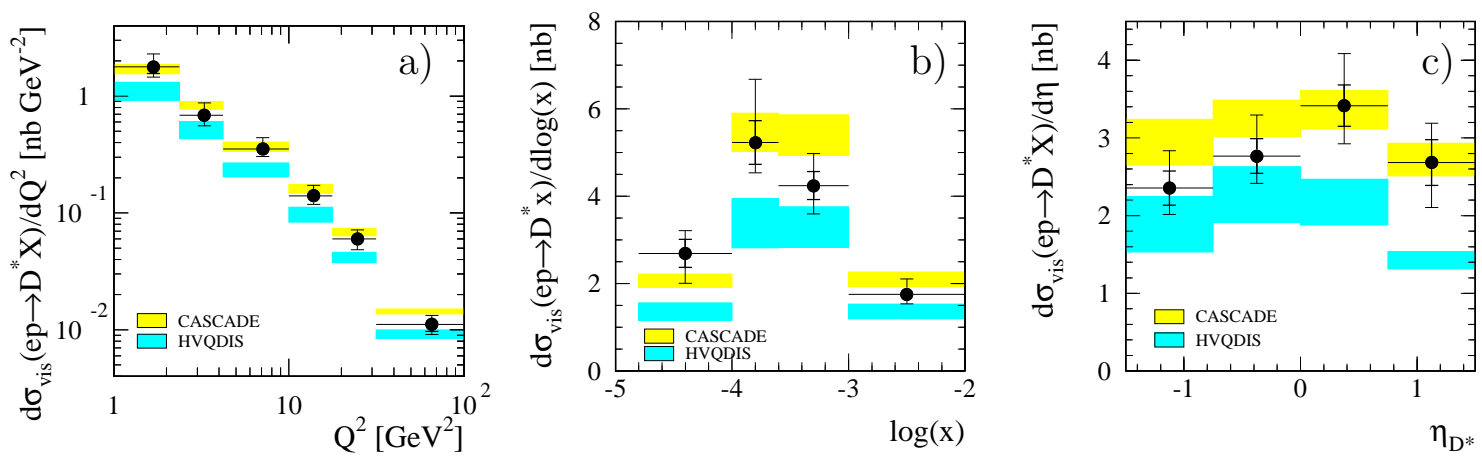

FIGURE 6. Single differential inclusive cross section $\sigma\left(e p \rightarrow e D^{*} X\right)$ as a function of a) $Q^{2}$, b) $x, c) \eta$. The expectations from HVQDIS (CASCADE) are indicated by the dark (light) shaded bands.

band) leading to a somewhat improved description of the $\eta^{D^{*}}$ distribution. Fig.6 shows the $\mathrm{H} 1$ data in comparison with the result from HVQDIS (dark shaded) and from CASCADE (light shaded). CASCADE describes the data fairly well in both normalization and shape while HVQDIS is lower and fails to describe the shape of the $\eta_{D^{*}}$-distribution in particular in the forward direction. At small values of $x$ CASCADE also provides a better description.

\section{EXTRACTION OF $F_{2}^{C \bar{C}}$}

The charm contribution to the proton structure function, $F_{2}^{c \bar{c}}$, is related to the charm cross section in $x$ and $Q^{2}$ as $\frac{d \sigma^{c \bar{c}}}{d x d Q^{2}}=\frac{2 \pi \alpha}{x Q^{4}}\left(1+(1-y)^{2}\right) \cdot F_{2}^{c \bar{c}}\left(x, Q^{2}\right)$. The contribution from $F_{L}$ is expected to be small and is neglected here. $F_{2}^{c \bar{c}}$ is extracted from the measured visible cross section in each bin of $x$ and $Q^{2}$ by extrapolation into the full phase space in $\eta$ and $p_{\perp}$ using the HVQDIS program. As shown in fig.7 there is good agreement between $\mathrm{H1}$ and ZEUS and, in general, with the prediction obtained from a NLO QCD fit to the inclusive $\mathrm{H} 1$ data. At the lowest $x$ and $Q^{2}$, a tendency towards a departure from the model is visible. Alternatively the CCFM model as implemented in CASCADE can be used for the extrapolation. Fig.7b) shows the comparison between the $\mathrm{H} 1$ data as extracted using CCFM and the CCFM expectation. There is good agreement also at the lowest values of $x$ and $Q^{2}$. A comparison of $F_{2}^{c \bar{c}}$ with the inclusive proton structure function $F_{2}$ shows that the contribution from charm to the inclusive cross section is larger than $10 \%$ everywhere in the region of $Q^{2}>1 \mathrm{GeV}^{2}$ and $x<10^{-3}$ and can be as large as $25 \%$ at large values of $Q^{2}$.

\section{OPEN BEAUTY PRODUCTION}

In contrast to charm quarks, $b$-quarks are much heavier, thus providing a harder scale for perturbative calculations. At the same time the total $b \bar{b}$ cross section is ex- 

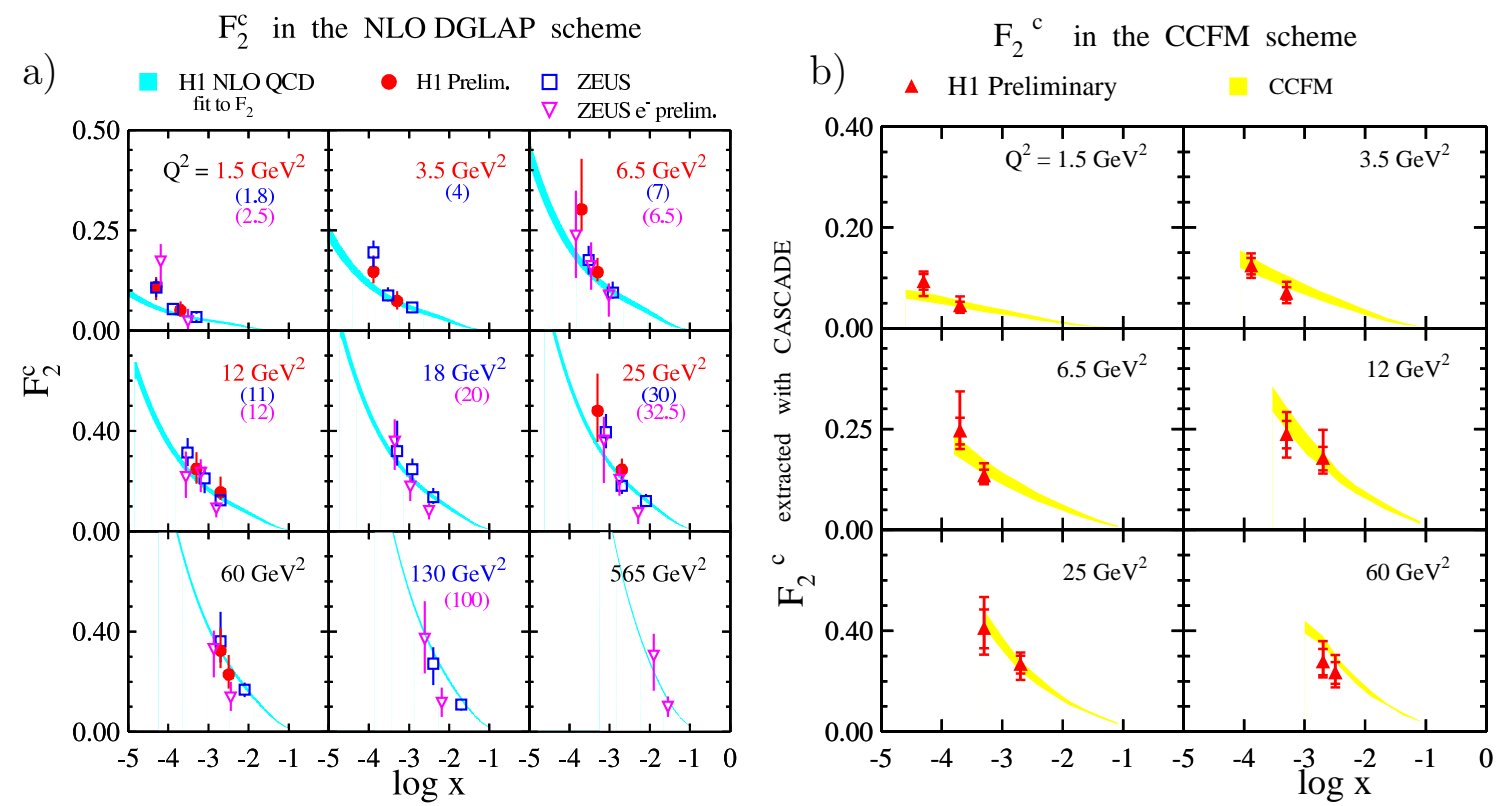

FIGURE 7. The charm contribution to the proton structure function $F_{2}^{c \bar{c}}$ as derived a) using the HVQDIS program (H1, ZEUS) b) using the CCFM model as implemented in CASCADE (H1). Also shown are the predictions for $F_{2}^{c \bar{c}}$ varying the charm quark mass between 1.3 and $1.5 \mathrm{GeV}$.

pected to be smaller than that of charm by a factor of 200 . The first measurements on the beauty production cross section in $e p$-scattering at HERA were performed using semi-leptonic decays $[10,12,11]$. In these analyses events with two or more jets are selected if they have at least one identified muon (electron) with a $p_{\perp}$ of 2 $\mathrm{GeV}(1.6 \mathrm{GeV})$ in the central region of the $\mathrm{H} 1$ (ZEUS) detector respectively. The lepton is assigned to one of the two jets of the event. To distinguish $b$-events from those with charm or fake leptons and to extract the $b \bar{b}$ cross section the transverse momentum $p_{T}^{r e l}$ of the lepton relative to the jet is used (fig.8a). Both measurements (fig.9) are above the NLO expectations.

Recently a new independent measurement has been presented by H1 [13]. The analysis uses the central silicon vertex detector to improve the track resolution. Events with $b$-mesons are tagged by their long lifetime using the impact parameter between the muon track and the beam in the transverse plane. From the impact parameter distribution (fig.8b) the $b \bar{b}$-cross section is extracted. A log-likelihood fit yields a value of $\sigma_{e p \rightarrow e b \bar{b} X}=(176 \pm 30$ (stat) \pm 29 (syst) $)$ pb which confirms the previous results. To maximize the $b$-contribution in the event sample and to reduce the systematics the two methods are combined in a likelihood fit (fig.10), yielding a cross section of $170 \pm 25 \mathrm{pb}$. Like the previous measurements this result is somewhat higher than the NLO QCD expectation [20] which gives $104 \pm 17 \mathrm{pb}$. 

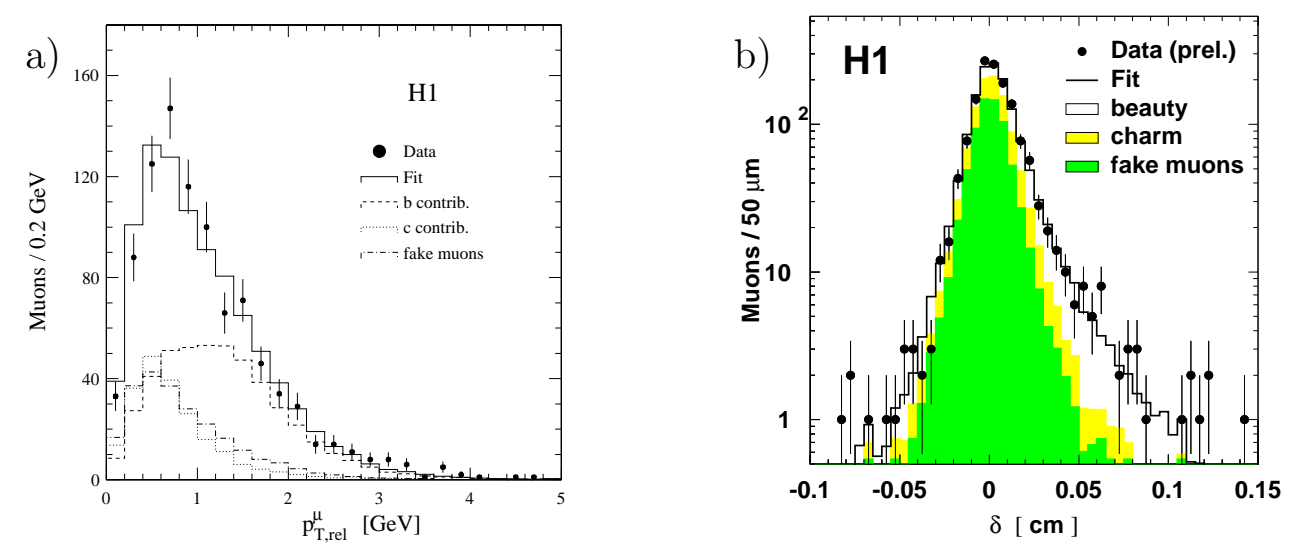

FIGURE 8. a) $p_{\perp, \text { rel }}^{\mu}$ of the data and the fitted sum (solid line) of the contributions from events with beauty, charm and fake muons. b) Impact parameter and decomposition from the likelihood fit.

\section{SUMMARY}

Since 1992 HERA has been collecting a wealth of data at continuously increasing luminosity. New results with charm and beauty data have been presented here. In the charm sector precision tests of QCD are being performed by confronting the data in a variety of channels $\left(D^{*}, D_{s}\right.$ and semi-leptonic decays) with predictions from NLO calculations. The process of boson gluon fusion has been shown to be by far the dominant contribution to the production of charm quarks in the wide kinematic range between the photoproduction regime, $Q^{2} \rightarrow 0$, and $Q^{2}<1000 \mathrm{GeV}^{2}$. However, in photoproduction, presently, the models do not fully reproduce the data in normalization and shape. In the region of low $Q^{2}\left(1<Q^{2}<600 \mathrm{GeV}^{2}\right)$ the charm contribution to the proton structure function $F_{2}^{c \bar{c}}$ has been extracted. The data have been compared to calculations using different parton evolution schemes and there are indications that the CCFM equation gives a better description of the data in the region of the smallest values of $x$. In the beauty sector a new systematically independent measurement using the lifetime tag (impact parameter) of semi-muonic events has been presented. The result confirms the previous measurements from H1 and ZEUS which are somewhat larger than expected by NLO calculations.

\section{ACKNOWLEDGMENTS}

I wish to thank the organizers for a very pleasant and fruitful meeting and my colleagues at ZEUS and H1 for supplying their data and the discussions. 


\section{ZEUS}
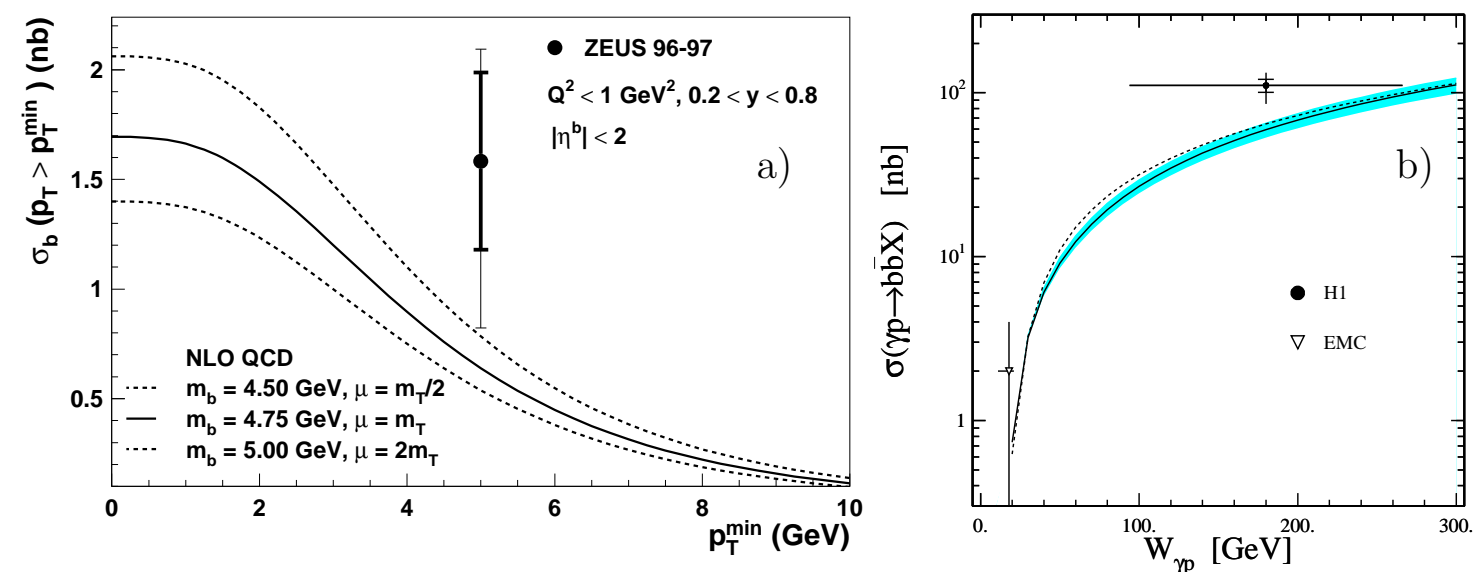

FIGURE 9. a) Extrapolated b photoproduction cross section as measured by the ZEUS Experiment for $p_{T}^{b}>p_{T}^{\min }=5 \mathrm{GeV}, 0.2<y<0.8$ and $\left|\eta^{b}\right|<2$ compared with NLO QCD predictions plotted as a function of $p_{T}^{\mathrm{min}}$. b) The total photoproduction cross section, $\sigma(\gamma p \rightarrow b \bar{b} X)$ measured by H1. The horizontal error bar represents the range of the measurement. The curve shows the expectation from the FMNR NLO calculation using different structure functions.

\section{REFERENCES}

1. B.W. Harris, J. Smith, Phys. Rev. D57 (1998) 2806.

2. V. N. Gribov and L. N. Lipatov, Sov. J. Nucl. Phys. 15 (1972) 438; L. N. Lipatov, Sov. J. Nucl. Phys. 20 (1975) 96.; G. Altarelli and G. Parisi, Nucl. Phys. B126 (1977) 298.

3. M. Ciafaloni, Nucl. Phys. B296 (1988) 49; S. Catani, F. Fiorani and G. Marchesini, Phys. Lett. B234 (1990) 339; Nucl. Phys. B336 (1990) 18.

4. H. Jung, Nucl. Phys. B (Proc. Suppl.) 79 (1999) 429; hep-ph/9905554.

5. H1 Coll., contr. paper ${ }^{2}$ to ICHEP2000, Osaka, 313.

6. ZEUS Coll., contr. paper to ICHEP2000, Osaka, 449.

7. ZEUS Coll., J. Breitweg et al., Eur. Phys. J. C12 (2000) 35.

8. ZEUS Coll., J. Breitweg et al., Phys. Lett. B481 (2000) 213.

9. ZEUS Coll., contr. paper to ICHEP2000, Osaka, 447.

10. H1 Coll., C. Adloff et al., Phys. Lett. B467 (1999) 156.

11. ZEUS Coll., contr. paper to IECHEP1999, Tampere, 498.

12. ZEUS Coll., J. Breitweg et al., DESY 00-166, accepted by Eur. Phys. J. C.

13. H1 Coll. contr. paper to ICHEP2000, Osaka, 311.

14. ZEUS Coll., contr. paper to ICHEP2000, Osaka, 448.

15. ZEUS Coll., J. Breitweg et al., Eur. Phys. J. C7 (1999) 609.

16. M. Glück, E. Reya, A. Vogt, Eur. Phys. J. C5 (1998) 461.

2) Contributed papers to International Conference on High Energy Physics, ICHEP2000, Osaka, July 2000. http://www-h1.desy.de/h1/www/publications/conf/list. ICHEP2000.html and http://www-zeus.desy.de/physics/phch/conf/osaka_paper.html 

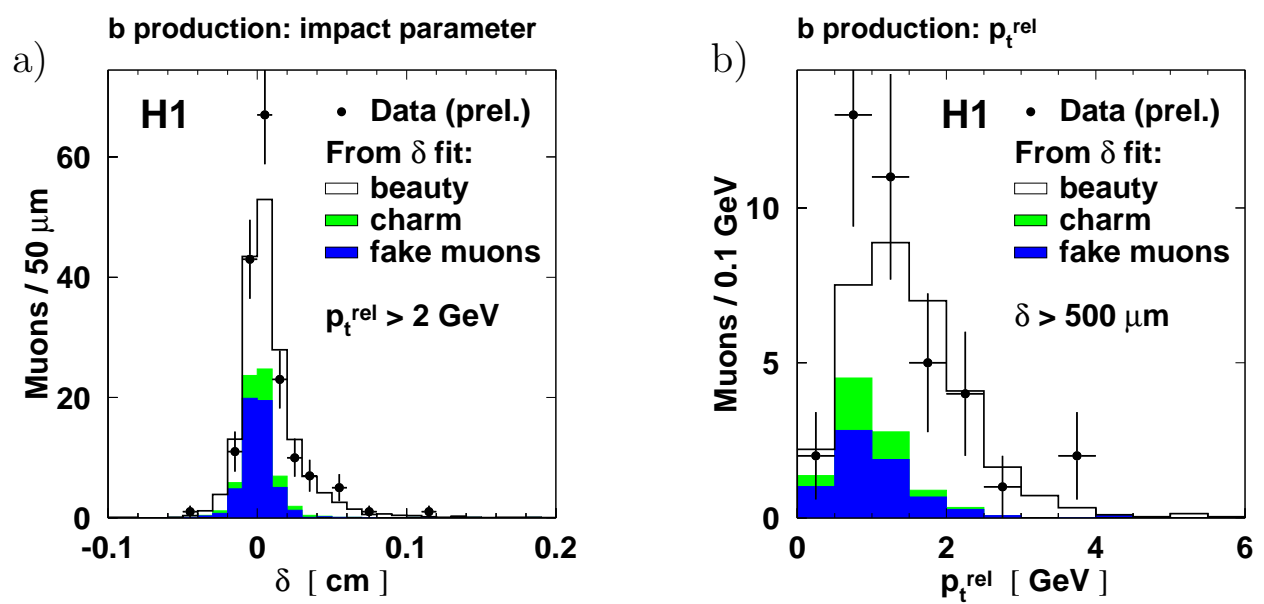

FIGURE 10. a) Impact parameter distribution for muon candidates with $p_{T}^{\text {rel }}>2 \mathrm{GeV}$ and b) $p_{T}^{r e l}$ distribution for muon candidates with $\delta>500 \mu m$, with estimated contributions.

17. H.L. Lai et al., Eur. Phys. J. C12 (2000) 375.

18. C. Peterson et al., Phys. Rev. D27 (1983) 105.

19. ZEUS Collab., J. Breitweg et al., Eur. Phys. J. C6 (1999) 67;

H1 Collab., C. Adloff et al., Z. Phys. C72 (1996) 593.

20. S. Frixione et al., Phys. Lett. B348, 633 (1995); Nucl. Phys. B454, 3 (1995).

21. E. Norrbin, T.Sjöstrand, hep-ph/9905493. Proc. of DESY Workshop "Monte Carlo Generators for HERA Physics".

22. ZEUS Coll., contr. paper to IECHEP1999, Tampere, 525.

23. L. Gladilin, hep-ex/9912064, 1999.

24. T.Sjöstrand, Comp. Phys. Commun. 82 (1994) 74.

25. H. Jung, Comp. Phys. Commun. 86, 147 (1995). 